\title{
A method to estimate the momentum and the decay proper time of the $B$ mesons from their hadronic and semileptonic decays
}

C. Defoix

Laboratoire de Physique Corpusculaire-College de France - Paris 
LPC $90-41$

\title{
A method to estimate \\ the momentum and the decay proper time of the $B$ mesons from their hadronic and semileptonic decays ${ }^{1}$.
}

\author{
Christian Defoix \\ Laboratoire de Physique Corpusculaire \\ Collège de France - Paris
}

June 28, 1990

\begin{abstract}
$\underline{\text { Abstract }}$
A method, which substitutes for the usual kinematical fit, is developed to estimate the momentum, and hence the decay proper time of the $B$ mesons produced in the $Z^{0}$ decay, in the experimental context of LEP. This method tries using the maximum information contained in the charged particle measurement and identification from such a device as DELPHI. Constrained and unconstrained final states involving a $D$ meson are treated in a similar way. Monte-Carlo simulation of the method shows that the resulting average uncertainty on the proper time, due to $B$ momentum estimation, reaches $7 \%$ for the hadronic decay events and $13 \%$ for the semileptonic ones, for half the events processed by the method in question.

From such results, analysis of topics such as the $B_{s}^{0}-\bar{B}_{s}^{0}$ oscillations can be reasonably envisaged at LEP.
\end{abstract}

${ }^{1}$ Work supported by IN2P3/CNRS - France 


\section{Introduction}

Progress in $B$ physics requires higher statistics of events and, for certain topics in this field, an accurate knowledge of the decay proper time, $t$, and hence of the momentum of the $B$. Among the topics in question, we may cite :

- estimation of differences between mean lifetimes of the various kinds of $B\left(B_{u}, B_{d}\right.$ and $B_{s}$ ), which can differ by a few percents.

- evidence for and characterization of the possibly high-frequency oscillations of the
$B_{s}^{0}$.

In the LEP context, which is mainly concerned by this paper, high luminosity and upgrade of the microvertex detector technique can be considered as important steps in this direction (Ref. [1]). Thus, the improved microvertex detector foreseen in the DEL$\mathrm{PHI}$ device increases the possibility of separating the successive vertices encountered in $B$ production and decay. An accuracy of $\sim 200 \mu \mathrm{m}$ is expected for the $B$ decay length $d$. The proper time $t$ being defined from $d$ and the $B$ momentum $P_{B}$ by the relationship :

$$
t=\frac{M_{B} d}{P_{B}}
$$
the mean relative contribution of the $d$ measurement to the global error on $t$, i.e. $\sigma_{t}$, is
reduced to $\sim 8 \%$.

Then the essential worry comes from the part of $\sigma_{t}$ which is due to the uncertainty

The $B \bar{B}$ system is produced in association with gluons from $Z^{0}$ decay and the $B$ momentum is spread according to the corresponding fragmentation function $f(z)$, where $z=P_{B} / E_{e}^{ \pm}$( $E_{e}^{ \pm}$being the beam energy at LEP). For instance the Lund generator used in the DELPHI simulation program foresees a $f(z)$ shape leading to a relative error on
$P_{B}$ :

$$
\frac{\sigma_{P_{B}}}{P_{B}} \simeq{ }_{-25}^{+16} \%
$$

Using only this function, without supplementary information on $P_{B}$, limits drastically the resulting precision on $t$. A possible approach to circumvent this problem, for the case of the $B$ semi-leptonic decays, is given in Ref. [2].

On the other hand, the usual kinematical fit, concerning only constrained events, leads to the needed precision but the event statistics, even at high luminosity of LEP, remains too small, particularly to analyse the $B_{s}^{0}$ oscillations (Ref. [1]).

The present paper describes a method of $P_{B}$ (or $t$ ) estimation which relies on the identification and measurement of the charged particles successively produced in the $B$ decay chains. Two dominant kinds of decays are considered, wich involve a $D$ meson in the final state :

1) hadronic $: B \rightarrow$ hadrons $+D$

2) semileptonic : $B \rightarrow l^{ \pm} \nu_{l}+D$ where $l^{ \pm}$is a $\mu^{ \pm}$or $e^{ \pm}$lepton. 
This method substitutes for the usual kinematical fit and results from an attempt to :

- increase the number of interesting events by a large order of magnitude with respect to the usual kinematical fit : constrained and unconstrained events are treated in a similar way and can be cumulated for a same physics goal.

- reach a precision on the $P_{B}$ (or $t$ ) estimation, for each event, which remains within the bounds required, for example, by the $B_{s}^{0}$ oscillation analysis.

It provides also a "normalized to 1" probability for each possible constrained or unconstrained final state of decay which can be envisaged for each event.

In the following, $B_{s}^{0}$ decays involving a $D_{s}^{ \pm}$decay, are chosen to illustrate the method. $D_{s}^{ \pm}$decay into at least three correctly measured charged particles is required, in order to ensure the minimal possibility of good $D$ vertex reconstruction.

In section 2 , the principle of the method is presented for the simplest case of $B$ hadronic decay :

$$
B_{s}^{0} \rightarrow \text { hadrons }+D_{s}^{ \pm} .
$$

As required for the $B_{s}^{0}$ oscillation analysis, and to minimize the wrong track-vertex assignments, the positions of $B$ production, and $B$ and $D$ decay vertices are assumed to be known and the latter well separated.

Various cases of hadronic decays into unconstrained, then constrained final states are described in section 3. The formulation for the other hadronic cases is carried out in Appendix A. Different estimations of $t$ are proposed and compared among them at the end of this section.

Section 4 discusses the case of the semileptonic decays, the $B$-decay vertex position of which is unknown. The particular cases corresponding to no or one particle unseen in the $D$ decay are explicitly treated in Appendix B.

In section 5 , the behaviour (robustness) of the chosen $t$ estimation is discussed in terms of bias and variance when :

- the possible perturbation coming from an unseen $\gamma$ issued from a $D_{s}^{*}$ decay is taken into account.

- an a priori knowledge of the $P_{B}$ distribution - for instance the fragmentation function - is introduced or not.

- the basic parameters used in the method are more or less wrong.

Conclusions end this paper in section 6 .

\section{Principle of the method.}

Let $k$ and $l$ (resp. $k^{\prime}$ and $l^{\prime}$ ) be the numbers of measured and unmeasured particles coming directly from the $B$ (resp. $D$ ) vertex. 
$\bar{q}_{B m}$ and $\bar{q}_{B 0}$ (resp. $\bar{q}_{D m}$ and $\bar{q}_{D 0}$ ) are the total energy-momentum 4-vectors of the $k$ (resp. $k^{\prime}$ ) measured and $l$ (resp. $l^{\prime}$ ) unmeasured particles at the $B$ (resp. $D$ ) vertex, $\bar{q}_{j}=\left[e_{j}, \vec{p}_{j}\right]$ being the 4 -momentum of each particle of mass $m_{j}$.

$\bar{Q}_{B}=\left[E_{B}, \vec{P}_{B}\right]$ and $\bar{Q}_{D}=\left[E_{D}, \vec{P}_{D}\right]$ are the unknown total 4-momenta respectively of the $B$ and $D$ mesons. One has :

$$
\bar{Q}_{B}=\bar{q}_{B m}+\bar{q}_{B 0} \text { with }\left(E_{B}^{2}-\left|\vec{P}_{B}\right|^{2}\right)^{1 / 2}=M_{B}
$$

and

$$
\bar{Q}_{D}=\bar{q}_{D m}+\bar{q}_{D 0} \text { with }\left(E_{D}^{2}-\left|\vec{P}_{D}\right|^{2}\right)^{1 / 2}=M_{D} .
$$

Notations are summarized on Fig. 1.

\subsection{Distribution of $P_{B}$, conditional to the 4-momenta measu- red at each decay vertex.}

Let $P\left\{\bar{q}_{B m}, \bar{q}_{D m}, P_{B}\right\}$ be the probability of observing the set of values $\left(\bar{q}_{B m}, \bar{q}_{D m}, P_{B}\right)$. In a first step, the conditional probability $P\left\{\left(\bar{q}_{B m}, \bar{q}_{D m}\right) / P_{B}\right\}$ of observing the values $\bar{q}_{B m}$ and $\bar{q}_{D m}$, given $P_{B}$, is formed. Then the probability $P\left\{P_{B} /\left(\bar{q}_{B m}, \bar{q}_{D m}\right)\right\}$ of observing the $P_{B}$ value, given $\bar{q}_{B m}$ and $\bar{q}_{D m}$, is deduced by inversion of the probabilities :

$$
\begin{aligned}
P\left\{\bar{q}_{B m}, \bar{q}_{D m}, P_{B}\right\} & =P\left\{\left(\bar{q}_{B m}, \bar{q}_{D m}\right) / P_{B}\right\} \times f\left(P_{B}\right) \\
& =P\left\{P_{B} /\left(\bar{q}_{B m}, \bar{q}_{D m}\right)\right\} \times P\left\{\bar{q}_{B m}, \bar{q}_{D m}\right\}
\end{aligned}
$$

where :

- $f\left(P_{B}\right)$ is the total probability of observing the value $P_{B}$ for the $B$ momentum magnitude ; in the following, it is likened to the a priori probability of $P_{B}$ : a flat distribution or, according to the degree of confidence one may have in it, an expression of the $B$ fragmentation function can be chosen (both possibilities have been tested, and compared). One assumes :

$$
\int_{P_{B \min }}^{P_{B \max }} f\left(P_{B}\right) d P_{B}=1
$$

- $P\left(\bar{q}_{B m}, \bar{q}_{D m}\right)$ in $\left(2^{\prime}\right)$ is the total probability of observing the pair of values $\left(\bar{q}_{B m}, \bar{q}_{D m}\right)$. It is independent of $P_{B}$.

Thus (2) and (2') lead to :

$$
P\left\{P_{B} /\left(\bar{q}_{B m}, \bar{q}_{D m}\right)\right\}=\frac{1}{C} \times P\left\{\left(\bar{q}_{B m}, \bar{q}_{D m}\right) / P_{B}\right\} \times f\left(P_{B}\right)
$$

where $C$ is a constant, independent of $P_{B}$ for a given event.

$F\left(P_{B}\right)=P\left\{P_{B} /\left(\bar{q}_{B m}, \bar{q}_{D m}\right)\right\}$ is the requested $P_{B}$ distribution from which the $B$ decay proper time distribution, $G(t)$, can be deduced :

$$
G(t)=\int F\left(P_{B}\right) \delta\left(t-\frac{M_{B} d}{P_{B}}\right) d P_{B}
$$


Except if needed, neither the errors on $\bar{q}_{B m}$ and $\bar{q}_{D m}$, nor any particular dependence of the matrix elements for the $B$ and $D$ decays, are taken into account, in order to simplify the presentation of this approach.

Then the conditional probability present in the r.h.s. of (3) is simply obtained by adding, for the considered event, the products of the mixing parameter $\gamma$ (homogeneous to a branching ratio) by the covariant phase space element $\Delta \varepsilon$ corresponding to each possible configuration of the set $\left(l, l^{\prime}\right)$ of unobserved particles (mainly : $\gamma, \pi^{0}, K_{L}^{0}, K_{S}^{0}\left(\rightarrow \pi^{0} \pi^{0}\right), \nu$, etc. . $):$

$$
P\left\{\left(\bar{q}_{B m}, \bar{q}_{D m}\right) / P_{B}\right\}=\sum_{l, l^{\prime}} \gamma_{k l} \gamma_{k^{\prime} l^{\prime}} \frac{\Delta \varepsilon_{k l k^{\prime} l^{\prime}}}{\varepsilon_{k l k^{\prime} l^{\prime}}^{\text {totall }}}
$$

- $\varepsilon_{k l k^{\prime} l^{\prime}}^{\text {total }}$ is the combined phase space weight for a $B$ meson decaying into the considered $k+l$ particles, plus a $D$ meson decaying in turn into the $k^{\prime}+l^{\prime}$ particles ; this weight has to be computed for all the $\bar{q}_{B m}$ and $\bar{q}_{D m}$ values accessible to measurement, for example in the DELPHI framework. Acceptance cuts on these quantities can prevent us from factorizing it according to the usual formula :

$$
\varepsilon_{k l k^{\prime} l^{\prime}}^{\text {total }}=\varepsilon_{k l}^{\text {total }} \times \varepsilon_{k^{\prime} l^{\prime}}^{\text {total }}
$$

- $\gamma_{k l}$ and $\gamma_{k^{\prime} l^{\prime}}$ are the corresponding mixing parameters, normalized to 1 for the $k$ and $k^{\prime}$ topologies (respectively for the $D$ and $B$ mesons) :

$$
\sum_{l} \gamma_{k l}=1 \quad ; \quad \sum_{l^{\prime}} \gamma_{k^{\prime} l^{\prime}}=1
$$

\subsection{Determination of the mixing parameters $\gamma_{k l}$ and $\gamma_{k^{\prime} l^{\prime}}$.}

In the present work, the mixing parameters are directly deduced from the $B$ and $D$ branching ratio values used in the simulation program. In practice, the $\gamma_{k l}$ or $\gamma_{k^{\prime} l^{\prime}}$ can be unknown, depend on the selection, mode of the events and have to be determined previously.

The integral of the r.h.s. of (5) over the all measurable values of $\bar{q}_{B m}$ and $\bar{q}_{D m}$ is equal to 1 for any value of $P_{B}$. Therefore, the formulation (5) can participate to form a likelihood function (l. f.), the optimisation of which leads to the $\gamma_{k l}$ and $\gamma_{k^{\prime} l^{\prime}}$ themselves, from the sample of the $N$ selected events.

The simplest formulation of this $1 . \mathrm{f}$. is :

$$
\mathcal{L}\left\{\bar{q}_{B m_{\nu}}, \bar{q}_{D m_{\nu}} ; \gamma_{k l}, \gamma_{k^{\prime} l^{\prime}}\right\}=\prod_{\nu=1}^{N} \int P\left\{\bar{q}_{B m \nu}, \bar{q}_{D m \nu}, P_{B}\right\} d P_{B}
$$

where the integrand is deduced from (2) and (5). In case of a good knowledge of the $B$ mean lifetime, $\tau_{B}$, the corresponding informative content can be introduced, leading to :

$$
\mathcal{L}=\prod_{\nu} \frac{1}{\mathcal{D}_{\nu}} \int_{t_{\min \nu}}^{t_{\max \nu}} \frac{e^{-t / \tau_{B}}}{\tau_{B}} P\left\{\bar{q}_{B m_{\nu}}, \bar{q}_{D m_{\nu}}, P_{B}\right\} \delta\left(P_{B}-\frac{M_{B} d_{\nu}}{t}\right) d t
$$

where : 
- $t_{\min \nu}$ and $t_{\max \nu}$ define the time bounds for observation of the $\nu^{\text {th }} B$ decay.

- $\mathcal{D}_{\nu}$ is the normalization integral :

$$
\mathcal{D}_{\nu}=\int_{t_{\min \nu}}^{t_{\max \nu}} \frac{e^{-t / \tau_{B}}}{\tau_{B}} \delta\left(P_{B}-\frac{M_{B} d_{\nu}}{t}\right) d t
$$

In each case, the mixing parameters are solutions of :

$$
\frac{\partial \mathcal{L}}{\partial \gamma_{k l}}=0 \quad ; \quad \frac{\partial \mathcal{L}}{\partial \gamma_{k^{\prime} l^{\prime}}}=0
$$

and the probabilities (5) are defined at the maximum of the 1 . f. associated to the concerned sample of events.

\section{Case of the hadronic $B$ decays : $B \rightarrow$ hadrons $+D$}

\subsection{General formulation}

At first sight, the calculation of $\Delta \varepsilon_{k l k^{\prime} l^{\prime}}$ in (5) could seem to be complex. Actually, successive trivial integrations over the unobserved variables lead to a final one-dimensional integral, at worse.

Using the factorization property of phase space, the general expression of $\Delta \varepsilon_{k l k^{\prime} l^{\prime}}$ can be written :

$$
\begin{aligned}
\Delta \varepsilon_{k l k^{\prime} l^{\prime}}= & \delta \varepsilon_{k^{\prime}} d^{4} \bar{q}_{D m} \int \delta \varepsilon_{l^{\prime}} \delta^{4}\left(\bar{Q}_{D}-\bar{q}_{D m}-\bar{q}_{D 0}\right) d^{4} \bar{q}_{D 0} \\
& \times \delta \varepsilon_{k} d^{4} \bar{q}_{B m} \int \delta \varepsilon_{l} \delta^{4}\left(\bar{Q}_{B}-\bar{Q}_{D}-\bar{q}_{B m}-\bar{q}_{B_{0}}\right) d^{4} \bar{q}_{B 0} d^{4} \bar{Q}_{D}
\end{aligned}
$$

where :

- the $B$ momentum is assumed to have a given value, $P_{B}$.

- the sign $\delta$ followed by an expression between parentheses represents the usual Dirac symbol.

- $\delta \varepsilon_{k} d^{4} \bar{q}_{B m}$ (resp. $\delta \varepsilon_{k^{\prime}} d^{4} \bar{q}_{D m}$ ) is the phase space element for the measured particles at the $B$ (resp. $D$ ) vertex, which is fixed for a given event and may be ignored : it does not modulate the final probability of $P_{B}$.

and :

$$
\begin{gathered}
\delta \varepsilon_{l}=\delta^{4}\left(\bar{q}_{B 0}-\sum_{j=1}^{l} \bar{q}_{j}\right) \prod_{j=1}^{l} \frac{d \vec{p}_{j}}{2 e_{j}} \\
\delta \varepsilon_{l^{\prime}}=\delta^{4}\left(\bar{q}_{D 0}-\sum_{j^{\prime}=1}^{l^{\prime}} \bar{q}_{j^{\prime}}\right) \prod_{j^{\prime}=1}^{l^{\prime}} \frac{d \vec{p}_{j^{\prime}}}{2 e_{j^{\prime}}}
\end{gathered}
$$

are the phase space elements for the $l$ and $l^{\prime}$ unmeasured particles, respectively at the $B$ and $D$ vertices. In the following $M_{B}^{0}$ and $M_{D}^{0}$ are the invariant masses of these sets of particles. 
The factors $\Delta \varepsilon_{k l k^{\prime} l^{\prime}}$ vary with $P_{B}$ and play an essential part to define the dependence on $P_{B}$ of the probabilities $P\left\{P_{B} / \bar{q}_{B m}, \bar{q}_{D m}\right\}$.

\subsection{Case of the unconstrained final states.}

\subsubsection{Case $l \geq 2$ and $l^{\prime} \geq 2$.}

Integrating in (7) over $d^{4} \bar{q}_{B 0}$ and $d^{4} \bar{q}_{D 0}$ and noting that the $D$ direction is fixed by measurement, we obtain :

$$
\Delta \varepsilon_{k l k^{\prime} l^{\prime}}=\int \varepsilon_{l}\left(M_{B}^{0}\right) \varepsilon_{l}^{\prime}\left(M_{D}^{0}\right) \frac{P_{D}^{2}}{2 E_{D}} d P_{D}
$$

where :

- $P_{D}=\left|\vec{P}_{D}\right|$

- $d \Omega_{D}$ is ignored in the element $d^{4} Q_{D}=\frac{P_{D}^{2}}{2 E_{D}} d P_{D} d \Omega_{D}$ because the $D$ direction is fixed.

- $M_{B}^{0}$ and $M_{D}^{0}$ depend on $P_{D}\left(\right.$ and $\left.P_{B}\right)$ :

$$
\begin{gathered}
M_{B}^{0}=\left[\left[\left(P_{B}^{2}+M_{B}^{2}\right)^{1 / 2}-E_{B m}-E_{D}\right]^{2}-\left|\vec{P}_{B}-\vec{P}_{B m}-\vec{P}_{D}\right|^{2}\right]^{1 / 2} \\
M_{D}^{0}=\left[\left(E_{D}-E_{D m}\right)^{2}-\left|\vec{P}_{D}-\vec{P}_{D m}\right|^{2}\right]^{1 / 2}
\end{gathered}
$$

- $\varepsilon_{l}\left(M_{B}^{0}\right)=\int d \varepsilon_{l}$ and $\varepsilon_{l^{\prime}}\left(M_{D}^{0}\right)=\int \delta \varepsilon_{l^{\prime}}$ are the total phase spaces for the $l$ and $l^{\prime}$ unmeasured particles.

The integration over $P_{D}$ has to be carried out in the physical region defined by :

$$
\begin{array}{rlrl}
E_{D m}+\sum_{j^{\prime}} m_{j^{\prime}} & \leq\left(M_{D}^{2}+P_{D}^{2}\right)^{1 / 2} & \leq\left(P_{B}^{2}+M_{B}^{2}\right)^{1 / 2}-E_{B m}-\sum_{j} m_{j} \\
\sum_{j} m_{j} & \leq \quad M_{B}^{0}: & \leq M_{B}-\left(E_{B m}^{2}-\left|\vec{P}_{B m}\right|^{2}\right)^{1 / 2} \\
\sum_{j^{\prime}} m_{j^{\prime}} & \leq & M_{D}^{0} & \leq M_{D}-\left(E_{D m}^{2}-\left|\vec{P}_{D m}\right|^{2}\right)^{1 / 2}
\end{array}
$$

Thus, one finds that the bounds of the physical region (9) are defined by a system of second order equations in $P_{D}$. These bounds limit substantially the possible values of $P_{B}$ leading to physical solutions. For example, for the decay chain :

$$
B^{0} \rightarrow \pi^{+} \pi^{-} \pi^{0} \pi^{0} D^{0}
$$

with $P_{B}=35 . \mathrm{GeV} / \mathrm{c}$ (mean value of $P_{B}$ at the $Z^{0}$ peak), one finds :

$$
\text { 23. } \mathrm{GeV} / \mathrm{c}<\mathrm{P}_{\mathrm{B}}<42 . \mathrm{GeV} / \mathrm{c}
$$

The calculation of $\varepsilon_{k l k^{\prime} l^{\prime}}^{\text {total }}$ results from a complete integration of (7) over all variables in the charged particles acceptance region. 


\subsubsection{Case $l=1$ and $l^{\prime} \geq 2$ (only one unmeasured particle at the $B$ vertex)}

In relation (7), the differential element $d^{4} \bar{q}_{B_{0}}$ becomes that of a particle with mass $m_{0}=\sqrt{e_{0}^{2}-\left|\vec{p}_{0}\right|^{2}}$, that is $: \frac{d \vec{p}_{0}}{2 e_{0}}$

Then $\Delta \varepsilon_{l k l^{\prime} k^{\prime}}$ takes the form :

$$
\Delta \varepsilon_{k 1 k^{\prime} l^{\prime}}=\int \varepsilon_{l^{\prime}}\left(M_{D}^{0}\right) \frac{P_{D}^{2}}{4 E_{D} e_{0}} \delta\left(g\left(P_{D}\right)\right) d P_{D}
$$

where :

$$
g\left(P_{D}\right)=\left(P_{B}^{2}+M_{B}^{2}\right)^{1 / 2}-E_{B m}-\left(\left|\vec{P}_{B}-\vec{P}_{B m}-\vec{P}_{D}\right|^{2}+m_{0}^{2}\right)^{1 / 2}-\left(P_{D}^{2}+M_{D}^{2}\right)^{1 / 2}
$$

$P_{D ; n}$ being those roots of the equation $g\left(P_{D}\right)=0$ which are in the physical region (9) (where $M_{B}^{0}=m_{0}$ ), one obtains :

$$
\Delta \varepsilon_{k 1 k^{\prime} l^{\prime}}=\sum_{n} \varepsilon_{l^{\prime}}\left(M_{D}^{0}\right) \frac{P_{D ; n}^{2}}{4 e_{0 ; n} E_{D ; n}} \frac{1}{\left|\frac{\partial g}{\partial P_{D}}\right|_{P_{D}=P_{D ; n}}}
$$

with :

$$
e_{0 ; n}=\left[\left|\vec{P}_{B}^{2}-\vec{P}_{B m}-\vec{P}_{D ; n}\right|^{2}+m_{0}^{2}\right]^{1 / 2}
$$

$g\left(P_{D}\right)=0$ is a second order equation having 0,1 or 2 roots in the physical region (9) according to the $P_{B}$ value its coefficients depend on.

The other unconstrained cases $\left(l \geq 2 ; l^{\prime}=1\right.$ and $l=l^{\prime}=1$; etc $\left.\ldots\right)$ are treated in Appendix A.

\subsection{Constrained final states}

In this case, the successive integrations over the unmeasured variables in (7) lead to the presence of residual $\delta$ functions. For example for $l=0$ and $l^{\prime} \geq 2$, the integrations over $P_{D}$ and $d^{4} \bar{q}_{D 0}$ give :

$$
\Delta \varepsilon_{k 0 k^{\prime} l^{\prime}}=\varepsilon_{l^{\prime}}\left(M_{D}^{0}\right) \frac{P_{D}}{2} \delta^{3}\left(\vec{P}_{B}-\vec{P}_{B m}-\vec{P}_{D}\right)
$$

where :

such that :

$$
\begin{aligned}
E_{D} & =\left(P_{B}^{2}+M_{B}^{2}\right)^{1 / 2}-E_{B m} \\
\left|\vec{P}_{D}\right| & =\left(E_{D}^{2}-M_{D}^{2}\right)^{1 / 2} \\
M_{D}^{0} & =\left[\left(E_{D}-E_{D m}\right)^{2}-\left|\vec{P}_{D}-\vec{P}_{D m}\right|^{2}\right]^{1 / 2}
\end{aligned}
$$

$$
\left\{\begin{array}{l}
E_{D} \geq M_{D} \\
\sum_{j^{\prime}=1}^{l^{\prime}} m_{j^{\prime}} \leq M_{D}^{0} \leq M_{D}-\left(E_{D m}^{2}-P_{D m}^{2}\right)^{1 / 2}
\end{array}\right.
$$

The presence of the factor $\delta^{3}$ in (10) can be solved by introducing the 3-dimensional distribution of the error on the total momentum $\vec{P}_{B m}$ of the particles measured at the 
$B$ vertex. The integral formed by the corresponding convolution product is trivial and
leads to :

$$
\Delta \varepsilon_{k 0 k^{\prime} l^{\prime}}=\varepsilon_{l^{\prime}}\left(M_{D}^{0}\right) \frac{P_{D}}{2} \prod_{\mu=1}^{3} \frac{1}{\sqrt{2 \pi} \sigma_{P_{B m}}^{\mu}} \exp \left\{-\frac{1}{2}\left(\frac{P_{B}^{\mu}-P_{D}^{\mu}-P_{B m}^{\mu}}{\sigma_{P_{B m}}^{\mu}}\right)^{2}\right\}
$$

The other cases, with $l=0$ and $l^{\prime}=1 ; l=l^{\prime}=0$; etc $\ldots$ are deferred to Appendix A.

\subsection{Results}

\subsubsection{Goodness of the results as a function of the number of unmeasured
particles}

$20,000 e^{+} e^{-} \rightarrow B \bar{B}+X$ events $\left(B=B_{u}, B_{d}\right.$ or $\left.B_{s}\right)$ have been simulated in DELPHI from the LUND generator used in the DELPHI simulation programs. The momentum and emission angles of each charged particle are randomly spread according to the multiple scattering in matter and the tracking accuracy provided by the DELPHI central detector and the upgraded version of the microvertex detector. The calculation of the covariant phase space elements $\Delta \varepsilon_{k l k^{\prime} l^{\prime}}$ and $\varepsilon_{k l k^{\prime} l^{\prime}}^{\text {total }}$ can take into account up to seven particles in the same set. Thus, up to $k=7$ charged and $l=7$ neutral particles can be considered at the $B$ vertex, in addition to the $D$ meson. The same possibilities exist for the $D$ meson decay. Within these limitations, for each current value of $P_{B}$, all purely hadronic, physically possible final states of the $B$ decays leading to a $D$ are taken into account. The results are considered directly in terms of $B$-decay proper time, the distribution of which results from relation (4). $\bar{t}$ being the "true" value of $t$, some typical distributions of $\frac{t-\bar{t}}{\bar{t}}$ obtained by the present method are shown as histograms on Fig. 2. The smooth curves, when drawn, include the mean error foreseen in DELPHI for the distance $d$ ( $8 \%$ for a $B$ mean decay length of $2.5 \mathrm{~mm}$ ).

The shaded zone of histograms corresponds to the part due to the final states which would be constrained in a usual kinematical fit.

One observes successively :

on Fig. 2a : the 2-constraint case, where all particles, except for the $D$ meson, are measured $(l=0)$. The part of the error due to the $P_{B}$ estimation, which is represented by the mean quadratic deviation $\sigma$, reaches $1.4 \%$ : its order of magnitude
is that given by a usual kinematical fit.

on Fig. 2b : an unconstrained case, only one $\pi^{0}$ being unmeasured at each vertex. The measured 4-momenta agree also with a final state where no missing particle is produced at the $B$ vertex (dashed zone). The total standard deviation is $\sigma \simeq 4.2 \%$ and the mean value of $\frac{t-\bar{t}}{\bar{t}}$ is -0.036 . Note the presence of two maxima.

on Figs. 2c and $\mathbf{d}$ : the number of missing particles is increased. The $t$ estimation is somewhat degraded but it remains acceptable for a $B_{s}^{0}-\bar{B}_{s}^{0}$ oscillation analysis
for example. 
on Fig. 2e : a total of seven particles are unmeasured. $\sigma$ reaches $24 \%$ and the bias on $t 27 \%$. The event is not interesting for the considered topics of physics.

As inferred by the previous observations, an event-by event goodness of the $t$ estimation can be introduced, which relies on the square root $\sigma$ of the variance of the $t$ distribution.

\subsubsection{Choice of the $t$ estimator}

Three kinds of $t$ estimators have been considered :

- the abscissa of the maximum of the $t$ distribution ; unfortunately this distribution, for a given event, can present two (or more) maxima of similar sizes, without possibility of choosing between them (particularly for the semileptonic decays).

- the $t$ distribution itself ; the mean distribution for the sample of $10,000 B \bar{B}$ events cited above, is drawn on Fig. 3a. Only the good events with $\sigma \leq 0.13$ participate to define this distribution. $\sim 55 \%$ of the $B_{s}^{0}$ decays with at least 3 charged particles at the $B$ and at the $D$ vertex are kept. The corresponding bias is compatible with 0 :

$$
\left\langle\frac{t-\bar{t}}{\bar{t}}\right\rangle \simeq 0.0046 \pm 0.0044
$$

whereas its mean quadratic deviation reaches :

$$
<\sigma>\simeq 0.11(=0.165 \text { before goodness cut })
$$

- the mean value $t_{\text {mean }}$, resulting from the $G(t)$ distribution for each event. The Monte-Carlo distribution of

$$
\frac{t_{\text {mean }}-\bar{t}}{\bar{t}}
$$

for the same $\sigma<0.13$ sample is presented on Fig. 3b. As expected, the corresponding bias equals the previous one and one obtains :

$$
<\sigma_{t_{\text {mean }}}>\simeq 0.07(=0.104 \text { before goodness cut })
$$

so that the latter is chosen as final $t$ estimator.

\section{Case of the semileptonic $B$ decays : $B \rightarrow l^{ \pm} \nu_{l}+D$}

\subsection{General formulation}

Only the positions of the main $A$ and $D$ vertices are supposed to be known. One assumes the $B$ decay vertex to be localized at an unknown abscissa $x$, on the charged lepton trajectory. The latter is locally taken as its tangent at the point of closest approach to the vertex $A$ (Fig. 4). One degree of freedom is added, and a lower accuracy is expected with respect to the hadronic decay cases.

The $a$ priori probability of $x$ is supposed to be flat. The only measured 4-momentum at the $B$ decay vertex is that of the lepton : $\bar{q}_{l_{m}^{ \pm}}$. 
Then the principle of probability inversion (2) has to be extended to the case of two random variables, in order to obtain the joint probability of $P_{B}$ and $x$, given the measured quantities $\bar{q}_{l_{m}^{ \pm}}$and $\bar{q}_{D m}$ :

$$
P\left\{\left(x, P_{B}\right) /\left(\bar{q}_{l_{m}^{ \pm}}, \bar{q}_{D_{m}}\right)\right\}=\frac{1}{C} P\left\{\left(\bar{q}_{l_{m}^{ \pm}}, \bar{q}_{D_{m}}\right) /\left(P_{B}, x\right)\right\} \times f\left(P_{B}\right)
$$

whence the $B$-decay proper time distribution can be deduced :

$$
G(t)=\int P\left\{\left(x, P_{B}\right) /\left(\bar{q}_{l_{m}^{ \pm}}, \bar{q}_{D_{m}}\right)\right\} \delta\left(t-\frac{M_{B}\left(x^{2}+\delta_{0}^{2}\right)^{1 / 2}}{P_{B}}\right) d P_{B} d x
$$

The conditional probability of $\bar{q}_{l_{m}^{ \pm}}$and $\bar{q}_{D_{m}}$, in (11), becomes :

$$
P\left\{\left(\bar{q}_{l_{m}^{ \pm}}, \bar{q}_{D_{m}}\right) /\left(P_{B}, x\right)\right\}=\sum_{k^{\prime}} \gamma_{k^{\prime} l^{\prime}} \frac{\Delta \varepsilon_{11 k^{\prime} l^{\prime}}}{\varepsilon_{11 k^{\prime} l^{\prime}}^{\text {total }}}
$$

where $k^{\prime}, l^{\prime}$ and $\gamma_{k^{\prime} l^{\prime}}$ are defined as previously for the $D$ decay vertex.

$\Delta \varepsilon_{11 k^{\prime} l^{\prime}}$ is the global phase space element for the successive decays :

$$
B \rightarrow l^{ \pm} \nu_{l}+D \text { and } D \rightarrow\left(k^{\prime}+l^{\prime}\right) \text { particles. }
$$

If needed, a decay matrix element can the introduced in the r.h.s. of (13). Presently, it is ignored to simplify the approach.

\subsection{Case $l^{\prime} \geq 2$}

From (7) one gets, using a self-explaining notation :

$$
\begin{aligned}
\Delta \varepsilon_{11 k^{\prime} l^{\prime}}= & \frac{d^{3} p_{l_{m}^{ \pm}}}{2 e_{l_{m}^{ \pm}}^{ \pm}} \int \delta\left(E_{B}-e_{l_{m}^{ \pm}}-\left|p_{\nu}\right|-E_{D}\right) \delta\left(\vec{P}_{B}-\vec{p}_{l_{m}^{ \pm}}-\vec{p}_{\nu}-\vec{P}_{D}\right) \frac{d^{3} p_{\nu}}{2\left|p_{\nu}\right|} \\
& \times \delta\left(\left(P_{D}^{2}+M_{D}^{2}\right)^{1 / 2}-E_{D 0}-E_{D m}\right) \delta\left(\vec{P}_{D}-\vec{P}_{D 0}-\vec{P}_{D m}\right) \\
& \times \varepsilon_{l^{\prime}}\left(M_{D}^{0}\right) d E_{D 0} d \vec{P}_{D 0} \frac{P_{D}^{2}}{2 E_{D}} d P_{D} d \Omega_{D}
\end{aligned}
$$

Integration over $\bar{q}_{D 0}$, then over $\vec{p}_{\nu}$ leads to :

$$
\Delta \varepsilon_{11 k^{\prime} l^{\prime}}=\frac{d^{3} p_{l_{m}^{ \pm}}}{2 e_{l_{m}^{ \pm}}^{ \pm}} d \Omega_{D} \int \frac{\varepsilon_{l^{\prime}}\left(M_{D}^{0}\right)}{2\left|\vec{P}_{B}-\vec{p}_{l_{m}^{ \pm}}-\vec{P}_{D}\right|} \delta\left(g\left(P_{D}\right)\right) \frac{P_{D}^{2}}{2 E_{D}} d P_{D}
$$

with :

- $M_{D}^{0}=\left[\left[\left(P_{D}^{2}+M_{D}^{2}\right)^{1 / 2}-E_{D m}\right]^{2}-\left|\vec{P}_{D}-\vec{P}_{D m}\right|^{2}\right]^{1 / 2}$

- $g\left(P_{D}\right)=\left(P_{B}^{2}+M_{B}^{2}\right)^{1 / 2}-e_{l_{m}^{ \pm}}-\left(P_{D}^{2}+M_{D}^{2}\right)^{1 / 2}-\left|\vec{P}_{B}-\vec{p}_{l_{m}}^{ \pm}-\vec{P}_{D}\right|$ 
- and the following definition of the physical bounds of integration :

$$
\left.\begin{array}{c}
E_{B}-E_{D}-e_{l_{m}^{ \pm}} \geq 0 \\
E_{D}-E_{D m} \geq \sum_{j^{\prime}=1}^{l^{\prime}} m_{j^{\prime}} \\
M_{D}-\left(E_{D m}^{2}-P_{D m}^{2}\right)^{1 / 2} \geq M_{D}^{0} \geq \sum_{j^{\prime}=1}^{l^{\prime}} m_{j^{\prime}}
\end{array}\right\}
$$

The equation $g\left(P_{D}\right)=0$ reduces to a second order equation in $P_{D}$ :

$$
A P_{D}^{2}+B P_{D}+C=0
$$

$A, B$ and $C$ are functions of $x$ and $P_{B}$, explicitly given in Appendix B.

This equation may have 0,1 or 2 roots, indexed by $n$, i.e. $P_{D ; n}$, in the physical region (15), for given values of $P_{B}$ and $x$. It appears that the $x$ values corresponding to physical solutions are restrained to an interval of a few hundreds of $\mu \mathrm{m}$, on average. On the other hand, the differential element $\frac{d^{3} p_{l_{m}^{ \pm}}}{2 e_{l_{m}}^{ \pm}} d \Omega_{D}$ can be ignored because of its negligible variations with $x$, and of its independence of $P_{B}$. One gets :

$$
\Delta \varepsilon_{11 l^{\prime} k^{\prime}} \simeq \sum_{n} \frac{1}{\left|\vec{P}_{B}-\vec{p}_{l_{m}^{ \pm}}-\vec{P}_{D}\right|} \frac{P_{D ; n}^{2}}{4 E_{D ; n}} \frac{\varepsilon_{l^{\prime}}\left(M_{D}^{0}\right)}{\left|\frac{\partial g}{\partial P_{D}}\right|_{P_{D}=P_{D ; n}}}
$$

where $E_{D ; n}=\left(P_{D ; n}^{2}+M_{D}^{2}\right)^{1 / 2}$.

The other cases $l^{\prime}=0$ and $l^{\prime}=1$ are treated in Appendix B.

\subsection{Results}

Various $G(t)$ distributions obtained from $B_{s}^{0}$ decays involving a $D_{s}^{ \pm}$decay which produces no (resp. one) unmeasured particle, are shown on Fig. 5 (resp. 6). Large differences of shape among events are found, which can be interpeted in the following way :

- For a given final state of $D_{s}$ decay, the energy-momentum conservation equations, which constrain strongly the shape of the factor $\Delta \varepsilon_{k l k^{\prime} l^{\prime}}$, admit physical solutions for two distinct sets of $\left(x, P_{B}\right)$ values, leading to split $G(t)$ distributions (Figs. $5 \mathrm{~b}, 5 \mathrm{c}, 6 \mathrm{c}$, and $6 \mathrm{~d}$ ). Often the sizes of the two parts are similar (Figs. 5b, 5c, $6 \mathrm{c}$ ), making impossible the determination of the good one. The two parts can be relatively distant from each other, or nearby, but forming a long tail, (Fig. 6b) and hence lead to a large variance.

- The compatibility of one same event with several final states involving different numbers of unmeasured particles in the $D_{s}$ decay can also lead to a long tail and a split distribution. This is the case on Fig. 6d, where the dashed zone represents the contribution of the hypothesis where no unmeasured particle has been produced. 
- We ignored in the present formulation the possibility of $D_{s}^{*}$ decay, with an unobserved $\gamma$. This fact can entail an appreciable shift of the $t$ distribution, as perceived on Figs. 5c or 6c for example.

As for the hadronic cases, we keep the good events, with $\sigma \leq .13$ only, so that $\sim 50 \%$ of the semileptonic events involving a $D_{s}$ decay into at least three measured particles, participate to get the average $\frac{t-\bar{t}}{\bar{t}}$ distribution on Fig. 7a. The mean bias :

$$
\left\langle\frac{t-\bar{t}}{\bar{t}}\right\rangle=-0.0064 \pm 0.01
$$

is compatible with 0 and the square root of the variance reaches :

$$
<\sigma>=0.16(=0.24 \text { before goodness cut). }
$$

On Fig. $7 \mathrm{~b}$, the distribution of $\frac{t_{\text {mean }}-\bar{t}}{\bar{t}}$ is shown, which leads to :

$$
<\sigma>=0.13(=0.17 \text { before goodness cut). }
$$

Note that this value of the quadratic mean deviation includes the largest part of the uncertainty on the $B$ vertex position, and hence on the $B$ decay length. The estimation which consists in taking the mean value $t_{\text {mean }}$ of $t$ on the $G(t)$ distribution remains the most efficient.

\section{Robustness of the $t$ estimator}

The quality of the previous estimator depends also on its sensitivity to possible perturbations and to the different possible formulations of a same basic hypothesis involved in the method.

\subsection{Dependence on the choice of the a priori probability of $P_{B}$}

Two extreme formulations of the probability $f\left(P_{B}\right) d P_{B}$ have been tested :

- a flat distribution on the range of possible values of $P_{B}:\left[0-E_{e^{ \pm}}\right]$.

- the fragmentation function provided by the Lund generator that is used to simulate the events $e^{+} e^{-} \rightarrow Z^{0} \rightarrow B \bar{B}+$ hadrons.

Relatively large difference are sometimes observed for a given event. But for the hadronic decays, the general characteristics, $\left\langle\frac{t_{\text {mean }}-\bar{t}}{\bar{t}}\right\rangle$ and $\langle\sigma\rangle$, of the average $G(t)$ distribution remain identical within 0.01 . 0.16 .

For the semileptonic decays, $\left\langle\frac{t_{\text {mean }}-\bar{t}}{\bar{t}}\right\rangle$ can reach $-0.04 ;\langle\sigma\rangle$ does not exceed

In each case, a flat $f\left(P_{B}\right)$ distribution provides the best results. 


\subsection{Effect of wrong mixing parameter values}

As already said, the parameters $\gamma_{k l}$ and $\gamma_{k^{\prime} l^{\prime}}$, in the whole present analysis, are directly calculated from the branching ratios of the various final states of $B_{s}^{0}$ and $D_{s}^{ \pm}$decays used for event simulation. Given the possible large misappreciation of these branching ratios, a different set of values has been randomly generated, corresponding to relative uncertainties up to $100 \%$, and introduced at the $t$ estimation level.

Conclusions are similar to the ones obtained for the previous test.

\subsection{Presence of an unseen photon coming from $D_{s}^{*}$ decay}

The Lund generator we used generates $75 \%$ of the $D_{s}$ produced in $B_{s}^{0}$ decay through $D_{s}^{*}$ decay :

$$
D_{s}^{*} \rightarrow D_{s}+\gamma
$$

Given this dominant origin for $D_{s}$, the introduction of the corresponding perturbation has been attempted in terms of $\gamma$ momentum distribution. The small $Q$-value (131 $\mathrm{MeV}$ ) of this $D_{s}^{*}$ decay leads us to approximate the $\gamma$ momentum distribution by that of its longitudinal component and the $D_{s}^{*}$ direction by that of the $D_{s}$.

The computer time is increased by an important factor, depending on the precision wanted to describe this $\gamma$ distribution. No significant improvement of the results is perceived, be it for the hadronic or the semileptonic case.

Thus the various causes of possible perturbation studied in the present work have no drastic consequence. As could be expected, the proposed estimators are essentially governed by the kinematical constraints forming, in relationships such as (7), the dominant structure of the phase space factors $\Delta \varepsilon_{k l k^{\prime} l^{\prime}}$.

Improvement could be brought nevertheless : for instance by detecting, in the electromagnetic calorimeters (and inside a restricted cone around the $D$ direction), the photon produced by the $D_{s}^{*}$, the dominance of which seems to be experimentally confirmed in $B_{s}^{0}$ decay. The measurement of the $K_{S}^{0}$ decays into $\pi^{+} \pi^{-}$, which are neglected here, would be a factor of improvement too.

\section{Conclusions}

Future $B$ physics at LEP, in the experimental frame of DELPHI, for example, requires at first high luminosity and a performant microvertex detector, allowing a good determination of the $z$ coordinate. The off-line data analysis has to take full advantage of these improvements to ensure the needed accuracy on the $B$-decay proper time - through a good knowledge of the $B$ momentum - while retaining sufficient statistics on the event samples needed for precise physics goals.

In the present paper, a method is proposed which attempts to reach this double aim. It relies on the measurement and identification of the charged particles produced at the $B$ and $D$ decay vertices. In any case the $D$ vertex is supposed to be correctly reconstructed. Constrained and unconstrained final states of $B$ decay, involving a $D$ 
meson decay, are treated according to the same principles. First applied to the hadronic events, the method can be extended to the semileptonic ones, the $B$ decay vertex of which has an a priori unknown position.

For each event :

- a $B$-decay proper time distribution of probability is formed, essentially from the phase space elements characterizing the various final states which are candidates to explain the event in question. It is shown that the corresponding mean value, $t_{\text {mean }}$, for the resulting distribution is the best estimation of the $B$ proper time.

- a goodness criterion is introduced, based on the variance of this distribution. It allows to select the events with a sufficient accuracy on $t$. A reasonable selection keeps $\sim 50 \%$ of the events having at least three measured particles at each vertex (which has also to be clearly defined).

In these conditions, the mean relative uncertainty on the time estimation, due to that on the $B$ momentum, reaches $\sim 7 \%$ for the hadronic decays and $\sim 13 \%$ for the semileptonic ones. For the latter, a large part of the error on the $B$ decay length is included.

The qualities of robustness of the proposed estimator are mainly determined by the constraints of energy-momentum conservation, which dominate in the expression of the phase space factors cited above.

For the $B$ hadronic decays, a misappreciation of the mixing parameters of the concerned final states, or in the formulation of the hypothetical shape of the a priori probability distribution of the $B$ momentum (fragmentation function) does not result in a significant increase of bias or statistical error. For the $B$ semileptonic decays, the proper time estimates are more sensitive to these factors, but their uncertainties remain within acceptable limits.

Thus, the method proposed in the present paper can lead to the accuracy and event sample sizes required by challenges such as bringing evidence for the expected highfrequency $B_{s}^{0}-\bar{B}_{s}^{0}$ time oscillations and determining their features.

\section{Acknowledgment}

I thank L. Behr for encouraging discussions about the approach presented in this paper. 


\section{Appendices}

\section{A Computation of the $P_{B}$ or $t$ probability distribu- tions for the hadronic decay cases}

A.a $g\left(P_{D}\right)=0$ equation $^{1}$ for $l=1$ and $l^{\prime} \geq 2$

The equation $g\left(P_{D}\right)=0$ amounts to :

$$
A P_{D}^{2}+B P_{D}+C=0
$$

$A, B$ and $C$ are the following functions of $P_{B}$ :

$$
\left.\begin{array}{l}
A\left(P_{B}\right)=4\left(\Delta E^{2}-\Delta P^{2}\right) \\
B\left(P_{B}\right)=-4 \Delta P S \\
C\left(P_{B}\right)=4 M_{D}^{2} \Delta E^{2}-S^{2}
\end{array}\right\}
$$

where :

$$
\begin{aligned}
\Delta E & =\sqrt{P_{B}^{2}+M_{B}^{2}}-E_{B m} \\
\Delta P & =P_{B} \cos \alpha_{B D}-P_{B m} \cos \alpha_{B D m} \\
S & =M_{B}^{2}+M_{D}^{2}+m_{0}^{2}-2 \sqrt{P_{B}^{2}+M_{B}^{2}} E_{B m}+2 P_{B} P_{B m} \cos \alpha_{B B m}
\end{aligned}
$$

$\alpha_{M N}$ is the angle between the $\vec{P}_{M}$ and $\vec{P}_{N}$ directions, $m_{0}$ is the mass of the only unmeasured particle at the $B$ vertex.

\section{A.b Case $l^{\prime}=1$ and $l \geq 2$ : only one unmeasured particle of mass $m_{0}^{\prime}$ at the $D$ decay vertex and more than one at the $B$ vertex.}

In the r.h.s. of relation (7), the element $d^{4} \bar{q}_{D 0}$, for the set of unmeasured particles at the $D$ vertex, has to be replaced by that of a defined mass particle : $\frac{d \vec{p}_{0}^{\prime}}{2 e_{0}^{\prime}}-$ with $m_{0}^{\prime}=\left(e_{0}^{\prime 2}-\left|\vec{p}_{0}^{\prime}\right|^{2}\right)^{1 / 2}$. The integration over this element in (7) leads to :

$$
\Delta \varepsilon_{k l k^{\prime} 1}=\sum_{n} \varepsilon\left(M_{B}^{0}\right) \frac{P_{D ; n}^{2}}{4 e_{0}^{\prime} E_{D ; n}} \frac{1}{\left|\frac{\partial h}{\partial P_{D}}\right|_{P_{D ; n}}}
$$

where :

- $h\left(P_{D}\right)=\left(P_{D}^{2}+M_{D}^{2}\right)^{1 / 2}-E_{D m}-\left(\left|\vec{P}_{D}-\vec{P}_{D m}\right|^{2}+m_{0}^{\prime 2}\right)^{1 / 2}$

- $P_{D ; n}$ are the physical roots of the equation $h\left(P_{D}\right)=0$.

- $E_{D ; n}=\left(P_{D ; n}^{2}+M_{D}^{2}\right)^{1 / 2}$

\footnotetext{
${ }^{1}$ See paragraph 3.2 .2 of text
} 
- $M_{B}^{0}=\left[\left(\left(P_{B}^{2}+M_{B}^{2}\right)^{1 / 2}-E_{B m}-E_{D ; n}\right)^{2}-\left|\vec{P}_{B}-\vec{P}_{B m}-\vec{P}_{D ; n}\right|^{2}\right]^{1 / 2}$

$h\left(P_{D}\right)=0$ reduces to a second order equation in $P_{D}$, the coefficients of which depend on the measured 4-momentum $\bar{q}_{D m}$ at the $D$ vertex :

$$
\alpha P_{D}^{2}+\beta P_{D}=\gamma=0
$$

with :

$$
\begin{aligned}
\alpha & =4\left(E_{D m}^{2}-P_{D m}^{2}\right) \\
\beta & =-4 S P_{D m} \cos \alpha_{B D m} \\
\gamma & =4 E_{D m}^{2} M_{D}^{2}-S^{2} \\
S & =M_{D}^{2}+E_{D M}^{2}-P_{D m}^{2}-m_{0}^{\prime 2}
\end{aligned}
$$

$\alpha_{B D m}$ is the angle between the $B$ direction and the total momentum $\vec{P}_{D m}$ of the particles measured at the $D$ decay vertex.

The physical roots $P_{D ; n}$ have to satisfy :

$$
\left.\begin{array}{rl}
E_{D m}+m_{0}^{\prime} & \leq M_{D}^{2}+P_{D ; n}^{2} \leq\left(P_{B}^{2}+M_{B}^{2}\right)^{1 / 2}-E_{B m}-\sum_{j=1}^{l} m_{j} \\
\sum_{j=1}^{l} m_{j} & \leq \quad M_{B}^{0} \quad \leq M_{B}-\left(E_{B m}^{2}-P_{B m}^{2}\right)^{1 / 2}
\end{array}\right\}
$$

\section{A.c Case $l=l^{\prime}=1$ : one unmeasured particle at each vertex}

$e_{0}$ and $m_{0}$ (resp. $e_{0}^{\prime}$ and $m_{0}^{\prime}$ ) being the energy and mass of the unmeasured particle at the $B$ (resp. $D$ ) vertex, one obtains after integrating over the corresponding one-particle phase space elements :

$$
\Delta \varepsilon_{k 1 k^{\prime} 1}=\int \frac{P_{D}^{2}}{8 e_{0} e_{0}^{\prime} E_{D}} \delta\left(g\left(P_{B}, P_{D}\right)\right) \delta\left(h\left(P_{D}\right)\right) d P_{D}
$$

where :

- $g\left(P_{B}, P_{D}\right)=\left(P_{B}^{2}+M_{B}^{2}\right)^{1 / 2}-E_{B m}-\left(P_{D}^{2}+M_{D}^{2}\right)^{1 / 2}-\left(\left|\vec{P}_{B}-\vec{P}_{B m}-\vec{P}_{D}\right|^{2}+m_{0}^{2}\right)^{1 / 2}$

- $h\left(P_{D}\right)=E_{D}-E_{D m}-\left(\left|\vec{P}_{D}-\vec{P}_{D m}\right|^{2}+m_{0}^{\prime 2}\right)^{1 / 2}$

The integration over $P_{D}$ leads to :

$$
\Delta \varepsilon_{k 1 k^{\prime} 1}=\sum_{n} \frac{P_{D ; n}^{2}}{8 e_{0 ; n} e_{0 ; n}^{\prime} E_{D ; n}} \frac{1}{\left|\frac{\partial h}{\partial P_{D}}\right|_{P_{D ; n}}} \delta\left(g\left(P_{B}, P_{D ; n}\right)\right)
$$

where $P_{D ; n}$ are the physical roots of the equation $h\left(P_{D}\right)=0$. The latter reduces to second order, similar to the previous one $(\beta)$. Using the change of variable (4) of 
text, $\Delta \varepsilon_{k 1 k^{\prime} 1}$, which is a function of $P_{B}$, becomes a function $\Delta G_{k 1 k^{\prime} 1}$ of $t$. Successive integrations over $P_{B}$ and $t$ lead to :

$$
\Delta G_{k 1 k^{\prime} 1}(t)=\sum_{n, n^{\prime}} \frac{P_{B ; n n^{\prime}} P_{D ; n}^{2}}{8 M_{B} e_{0 ; n}^{\prime} e_{0 ; n n^{\prime}} E_{D ; n}} \frac{1}{\left|\frac{\partial h}{\partial P_{D}}\right|_{P_{D ; n}}} \frac{1}{\left|\frac{\partial g}{\partial P_{B}}\right|_{P_{D ; n}, P_{B ; n n^{\prime}}}} \mathcal{G}\left(\frac{P_{B n^{\prime} n} t}{M_{B}}, d, \sigma_{d}\right)
$$

where :

- $P_{B ; n n^{\prime}}$ are those roots of the equation in $P_{B}: g\left(P_{B}, P_{D ; n}\right)=0$, which are located in the physical region.

- $e_{0 ; n n^{\prime}}$ depends on $P_{D ; n}$ and $P_{B ; n n^{\prime}}$ :

$$
e_{0 ; n n^{\prime}}=\left(P_{B ; n n^{\prime}}^{2}+M_{B}^{2}\right)^{1 / 2}-E_{B m}-\left(P_{D ; n}^{2}+M_{D}^{2}\right)^{1 / 2}
$$

and $e_{0 ; n}^{\prime}$ exclusively on $P_{D ; n}$ :

$$
e_{0 ; n}^{\prime}=\left(P_{D ; n}^{2}+M_{D}^{2}\right)^{1 / 2}-E_{D m}
$$

- $\mathcal{G}$ represents the distribution of error, supposed to be gaussian :

$$
\mathcal{G}\left(w, \bar{w}, \sigma_{w}\right)=\frac{1}{\sqrt{2 \pi} \sigma_{w}} \exp \left\{-\frac{1}{2}\left(\frac{w-\bar{w}}{\sigma_{w}}\right)^{2}\right\}
$$

After reduction, the equation $g=0$ takes the form :

$$
A_{n} P_{B}^{2}+B_{n} P_{B}+C_{n}=0
$$

$A_{n}, B_{n}$ and $C_{n}$ being the following functions of $P_{D ; n}$ :

$$
\begin{aligned}
& A_{n}=4\left(\Delta E_{n}^{2}-\Delta P_{n}^{2}\right) \\
& B_{n}=-4 \Delta P_{n} S_{n} \\
& C_{n}=4 M_{B}^{2} \Delta E_{n}^{2}-S_{n}^{2}
\end{aligned}
$$

with :

$$
\begin{aligned}
\Delta E_{n} & =E_{B m}+E_{D ; n} \\
\Delta P_{n} & =P_{B m} \cos \alpha_{B B_{m}}+P_{D ; n} \cos \alpha_{B D} \\
S_{n} & =M_{B}^{2}+M_{D}^{2}+E_{B m}^{2}-P_{B m}^{2}-m_{0}^{2}+2 E_{B m} E_{D ; n}-2 P_{B m} P_{D ; n} \cos \alpha_{D B m}
\end{aligned}
$$

Thus, in this case, $\Delta G(t)$ depends on $t$ uniquely through a sum of gaussian functions.

\section{A.d Case $l=0, l^{\prime}=1$ : no unmeasured particle at the $B$ vertex and one at the $D$ vertex}

One obtains in this case :

$$
\Delta G_{k 0 k^{\prime} 1}(t)=\sum_{n^{\prime}} \frac{P_{B ; n^{\prime}} P_{D}}{4 M_{B} e_{0}^{\prime}} \mathcal{G}_{B d}^{n^{\prime}}
$$

where : 
- $P_{D}=\left[\left(E_{B}-E_{B m}\right)^{2}-M_{D}^{2}\right]^{1 / 2}$

- $e_{0}^{\prime}=\left(P_{D}^{2}+M_{D}^{2}\right)^{1 / 2}-E_{D m}$

- $P_{B ; n^{\prime}}$ are the physical roots of the equation in $P_{B}$ :

$$
g\left(P_{B}, P_{D}\right)=\left(P_{B}^{2}+M_{B}^{2}\right)^{1 / 2}-E_{B m}-E_{D}-\left(\left|\vec{P}_{D}-\vec{P}_{D m}\right|^{2}+m_{0}^{2}\right)^{1 / 2}=0
$$

- $\mathcal{G}_{B d}^{n^{\prime}}$ includes the distributions of error for the $\vec{P}_{B m}$ and $d$ measurements, which depend on $\vec{P}_{B ; n^{\prime}}$ and $\vec{P}_{D}$ :

$$
\mathcal{G}_{B d}^{n^{\prime}}=\mathcal{G}\left(\frac{P_{B ; n^{\prime}} t}{M_{B}}, d, \sigma_{d}\right) \prod_{\mu=1}^{3} \mathcal{G}\left(P_{B ; n^{\prime}}^{\mu}-P_{D}^{\mu}, P_{B m}^{\mu}, \sigma_{P_{B m}}^{\mu}\right)
$$

according to the notation introduced above.

The resulting $t$ distribution is also a sum of gaussian functions of $t$.

\section{A.e Case $l \geq 2$ and $l^{\prime}=0:$ no unmeasured particle at the $D$ decay vertex.}

$$
\Delta \varepsilon_{k l k^{\prime} 0}=\left(E_{D m}^{2}-M_{D}^{2}\right) \varepsilon_{l}\left(M_{B}^{0}\right) \prod_{\mu=1}^{3} \mathcal{G}\left(P_{D}^{\mu}, P_{D m}^{\mu}, \sigma_{P_{D m}}^{\mu}\right)
$$

with :

- $P_{D}=\left(E_{D m}^{2}-M_{D}^{2}\right)^{1 / 2}$

- $M_{B}^{0}=\left[\left(\left(P_{B}^{2}+M_{B}^{2}\right)^{1 / 2}-E_{B m}-E_{D m}\right)^{2}-\left(\vec{P}_{B}-\vec{P}_{B m}-\vec{P}_{D}\right)^{2}\right]^{1 / 2}$

- $\prod_{\mu=1}^{3} \mathcal{G}$ is the error distribution for the $\vec{P}_{D m}$ measurement.

\section{A.f Case $l=1$ and $l^{\prime}=0$}

where :

$$
\Delta G_{k 1 k^{\prime} 0}(t)=\sum_{n^{\prime}} \frac{P_{D} P_{B ; n^{\prime}}}{4 M_{B} e_{0 ; n^{\prime}}} \frac{1}{\left|\frac{\partial g}{\partial P_{B}}\right|_{P_{B ; n^{\prime}}}} \mathcal{G}_{D d}^{n^{\prime}}
$$

- $P_{D}=\left(E_{D m}^{2}-M_{D}^{2}\right)^{1 / 2}$

- $P_{B ; n^{\prime}}$ are the physical roots of the equation in $P_{B}$ :

$$
g\left(P_{B}, P_{D}\right)=\left(P_{B}^{2}+M_{B}^{2}\right)^{1 / 2}-E_{B m}-\left(P_{D}^{2}+M_{D}^{2}\right)^{1 / 2}-\left(\left|\vec{P}_{B}-\vec{P}_{B m}-\vec{P}_{D}\right|^{2}+m_{0}^{2}\right)^{1 / 2}=0
$$


- $e_{0 ; n^{\prime}}=\left(P_{B ; n^{\prime}}^{2}+M_{B}^{2}\right)^{1 / 2}-E_{B m}-E_{D m}$

- $\mathcal{G}_{D d}^{n^{\prime}}$ includes the distributions of error for the $\vec{P}_{D m}$ and $d$ measurements, which depend on $\vec{P}_{D}$ and $P_{B ; n^{\prime}}$ :

$$
\mathcal{G}_{D d}^{n^{\prime}}=\mathcal{G}\left(\frac{P_{B ; n^{\prime}} t}{M_{B}}, d, \sigma_{d}\right) \prod_{\mu=1}^{3} \mathcal{G}\left(P_{D}^{\mu}, P_{D m}^{\mu}, \sigma_{P_{D m}}^{\mu}\right)
$$

\section{B Computation of the $t$ probability distribution for the semileptonic cases}

B.a Equation $g\left(P_{D}\right)=0$ for $l^{\prime} \geq 2$

$P_{B}$ and $x$ being fixed, $g\left(P_{D}\right)=0$ reduces to :

$$
A\left(P_{B}, x\right) P_{D}^{2}+B\left(P_{B}, x\right) P_{D}+C\left(P_{B}, x\right)=0
$$

where :

$$
\begin{aligned}
& A\left(P_{B}, x\right)=4\left(\Delta E^{2}-\Delta P^{2}\right) \\
& B\left(P_{B}, x\right)=-4 \Delta P S \\
& C\left(P_{B}, x\right)=4 M_{D}^{2} \Delta E^{2}-S^{2}
\end{aligned}
$$

and :

$$
\begin{aligned}
\Delta E & =\left(P_{B}^{2}+M_{B}^{2}\right)^{1 / 2}-e_{l_{m}^{ \pm}} \\
\Delta P & =P_{B} \cos \alpha_{B D}-p_{l_{m}^{ \pm}} \cos \alpha_{D l_{m}^{ \pm}} \\
S & =M_{B}^{2}+M_{D}^{2}-2\left(P_{B}^{2}+M_{B}^{2}\right)^{1 / 2} e_{l_{m}^{ \pm}}+2 P_{B} p_{l_{m}^{ \pm}} \cos \alpha_{B l_{m}^{ \pm}}
\end{aligned}
$$

The dependence on $x$ is implicit through the angles $\alpha_{M N}$, defined as in Appendix A.a.

\section{B.b Case $l^{\prime}=1$ : only one unmeasured particle of mass $M_{0}$ at the $D$ vertex}

From relation (14), where the element $d E_{0} d \vec{P}_{0}$ has to be replaced by that of a definedmass particle, $\frac{d \vec{p}_{0}}{2 e_{0}}$ with :

$$
m_{0}=\left(e_{0}^{2}-\left|\vec{p}_{0}\right|^{2}\right)^{1 / 2}
$$

one gets :

where :

$$
\Delta \varepsilon_{11 k^{\prime} 1}=\sum_{n} \frac{P_{D ; n}^{2}}{8\left|p_{\nu ; n}\right| e_{0 ; n} E_{D ; n}} \frac{1}{\left|\frac{\partial h}{\partial P_{D}}\right|_{P_{D ; n}}} \delta\left(g\left(P_{B}, P_{D ; n}\right)\right)
$$

- $P_{D ; n}$ are the physical roots of the equation :

$$
h\left(P_{D}\right)=\left(P_{D}^{2}+M_{D}^{2}\right)^{1 / 2}-E_{D m}-\left(\left(\vec{P}_{D}-\vec{P}_{D m}\right)^{2}+m_{0}^{2}\right)^{1 / 2}=0
$$


- $\left|p_{\nu ; n}\right|$ is the neutrino energy for $\vec{P}_{D}=\vec{P}_{D ; n}$ :

$$
\left|p_{\nu ; n}\right|=\left|\vec{P}_{B}-\vec{p}_{l_{m}^{ \pm}}-\vec{P}_{D ; n}\right|
$$

- $g\left(P_{B}, P_{D ; n}\right)=\left(P_{B}^{2}+M_{B}^{2}\right)^{1 / 2}-e_{l_{m}^{ \pm}}-E_{D ; n}-\left|p_{\nu ; n}\right|$

$P_{B ; n n^{\prime}}$ being the physical roots of the equation $g\left(P_{B}, P_{D ; n}\right)=0$, one gets from relation (4), after integrating over $P_{B}$ and $x$ :

$$
\Delta G_{11 k^{\prime} 1}(t)=\sum_{n^{\prime}, n} \frac{P_{D ; n}}{8\left|p_{\nu ; n}\right| e_{0 ; n} E_{D ; n}} \frac{1}{\left|\frac{\partial h}{\partial P_{D}}\right|_{P_{D ; n}}} \frac{1}{\left|\frac{\partial g}{\partial P_{B}}\right|_{P_{D ; n}, P_{B ; n n^{\prime}}}} \frac{1}{\left|\frac{\partial t}{\partial x}\right|_{P_{B ; n n^{\prime}}}}
$$

where

$$
\left|\frac{\partial t}{\partial x}\right|_{P_{B ; n n^{\prime}}}=\frac{P_{B ; n n^{\prime}}}{M_{B}}\left(1-\frac{\delta_{0}^{2} P_{B ; n n^{\prime}}^{2}}{M_{B}^{2} t^{2}}\right)^{1 / 2}
$$

expresses the form of the $t$ dependence.

B.c Case $l^{\prime}=0$ : no unmeasured particle at the $D$ vertex. In a first step, one gets :

$$
\Delta \varepsilon_{11 k^{\prime} 0}=\frac{P_{D}}{4\left|p_{\nu}\right|} \delta\left(g\left(P_{B}, P_{D}\right)\right) \delta^{3}\left(\vec{P}_{D}-\vec{P}_{D m}\right)
$$

where :

- $P_{D}=\left(E_{D m}^{2}-M_{D}^{2}\right)$

- $\left|p_{\nu}\right|=\left|\vec{P}_{B}-\vec{p}_{l_{m}^{ \pm}}-\vec{P}_{D}\right|$

- $g\left(P_{B}, P_{D}\right)=\left(P_{B}^{2}+M_{B}^{2}\right)^{1 / 2}-e_{l_{m}^{ \pm}}-E_{D m}-\left|p_{\nu}\right|$

Let $P_{B ; n}$ be the physical roots of the equation in $P_{B}: g\left(P_{B}, P_{D}\right)=0$. After introducing the experimental resolution on the three components of $\vec{P}_{D m}$ and integrating over $x$, one obtains :

$$
\Delta G_{11 k^{\prime} 0}(t)=\sum_{n} \frac{M_{B} P_{D}}{4\left|p_{\nu ; n}\right| P_{B ; n}} \frac{1}{\left|\frac{\partial g}{\partial P_{B}}\right|_{P_{D}, P_{B ; n}}}\left(1-\frac{\delta_{0}^{2} P_{B ; n}^{2}}{M_{B}^{2} t^{2}}\right)^{-1 / 2} \prod_{\mu} \mathcal{G}\left(P_{D}^{\mu}, P_{D m}^{\mu}, \sigma_{P_{D m}}^{\mu}\right)
$$

Thus, in contradistinction with the case $l^{\prime} \geq 2$, where a continuum of $P_{B}$ values is possible for a given value of $x$, for $l^{\prime} \leq 1$, only a discrete set of $n_{l^{\prime}}$ values of $P_{B}$ can be envisaged for each value of $x$, with $n_{l^{\prime}} \leq 2+2 l^{\prime}$. 


\section{Figure Captions}

Fig. 1 : Schema of the $B$ hadronic decay in terms of the four-momenta involved in text.

Fig. 2 : $G(t)$ distribution, normalized to 1, obtained for five typical cases of $B_{s}^{0}$ hadronic decay.

Fig. 3 : Hadronic decays ; from a sample of $10,000 Z^{0} \rightarrow B \bar{B}$ events $\left(B=B_{u}, B_{d}\right.$ and $\left.B_{s}\right)$ and after goodness selection $\sigma(\leq 0.13)$ :

a) average $G(t)$ distribution of the variable $\frac{t-\bar{t}}{\bar{t}}$.

b) distribution of the variable $\frac{t_{\text {mean }}-\bar{t}}{\bar{t}}$.

Fig. 4 : Schema of the $B$ semileptonic decay in terms of the four-momenta involved in text.

Fig. 5 : $G(t)$ distribution, normalized to 1 , obtained for three typical $B_{s}^{0}$ semileptonic decays, all particles produced in the $D_{s}^{ \pm}$decay being measured.

Fig. $6: G(t)$ distribution, normalized to 1 , for five typical $B_{s}^{0}$ semileptonic decays, the $D_{s}^{ \pm}$decay involving one unmeasured particle.

Fig. 7 : Semileptonic decays ; from the sample and the selection defined in the text :
a) average $G(t)$ distribution of the variable $\frac{t-\bar{t}}{\bar{t}}$.
b) distribution of $\frac{t_{\text {mean }}-\bar{t}}{\bar{t}}$. 


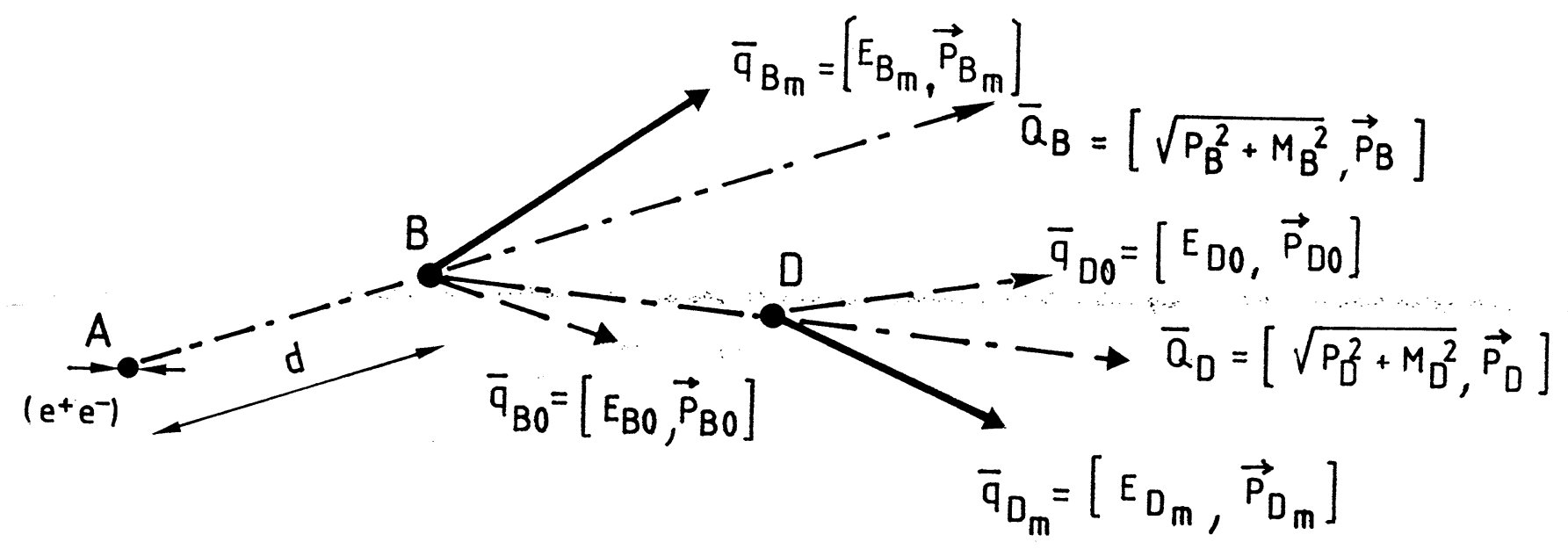

Fig. 1 


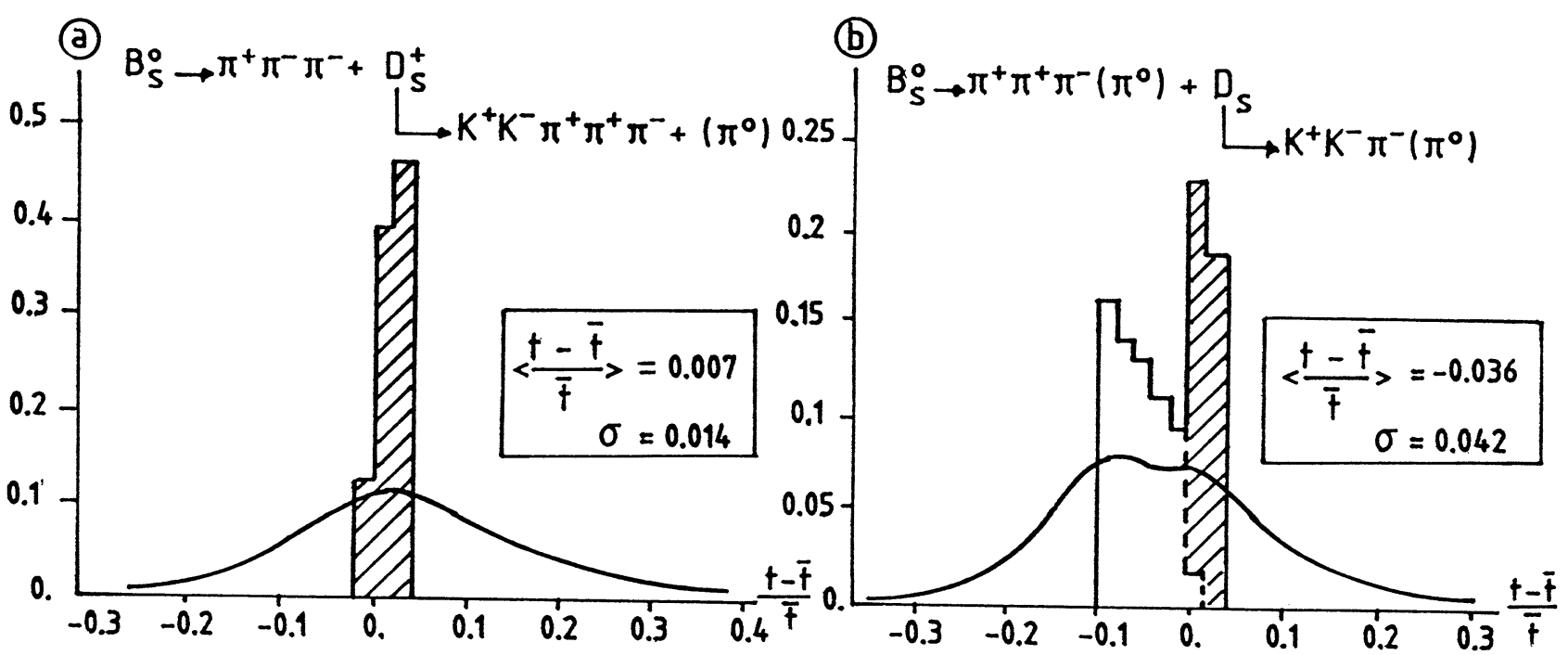

(c)

(d)

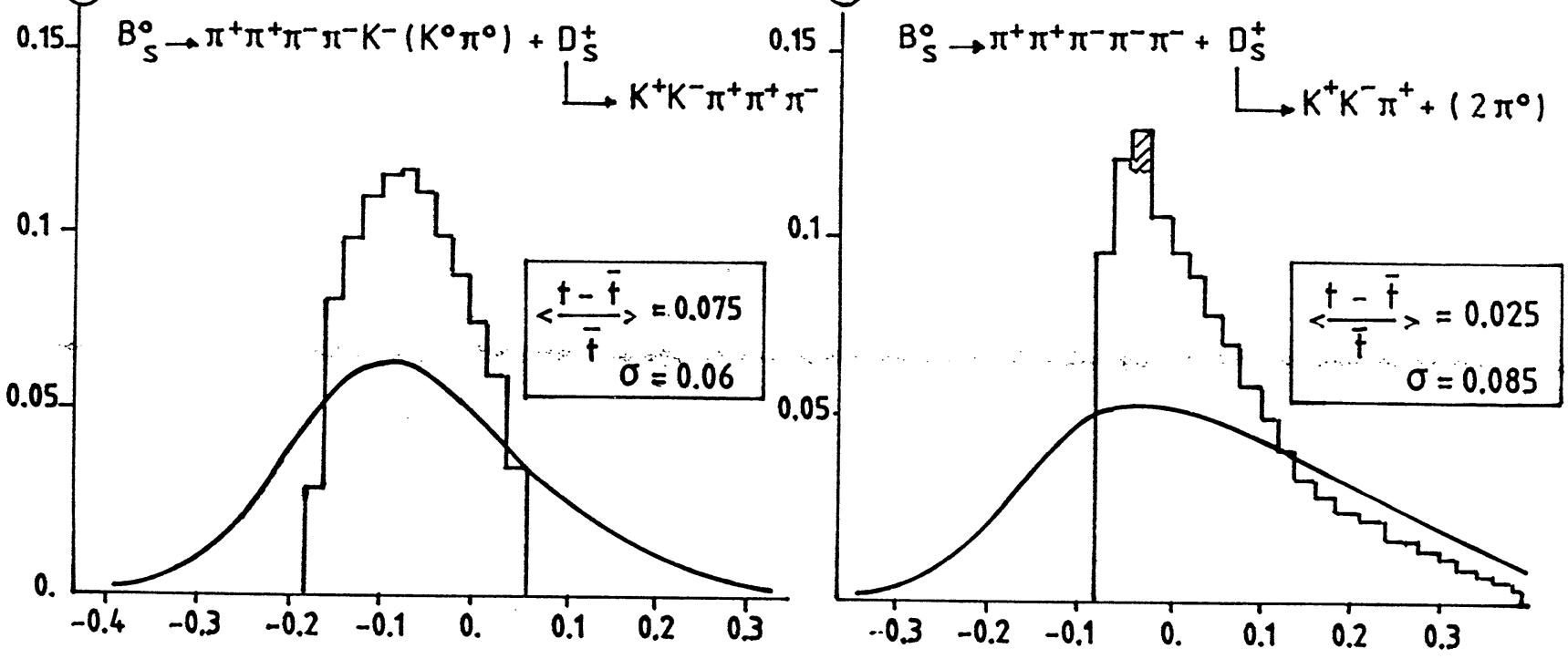

(e)

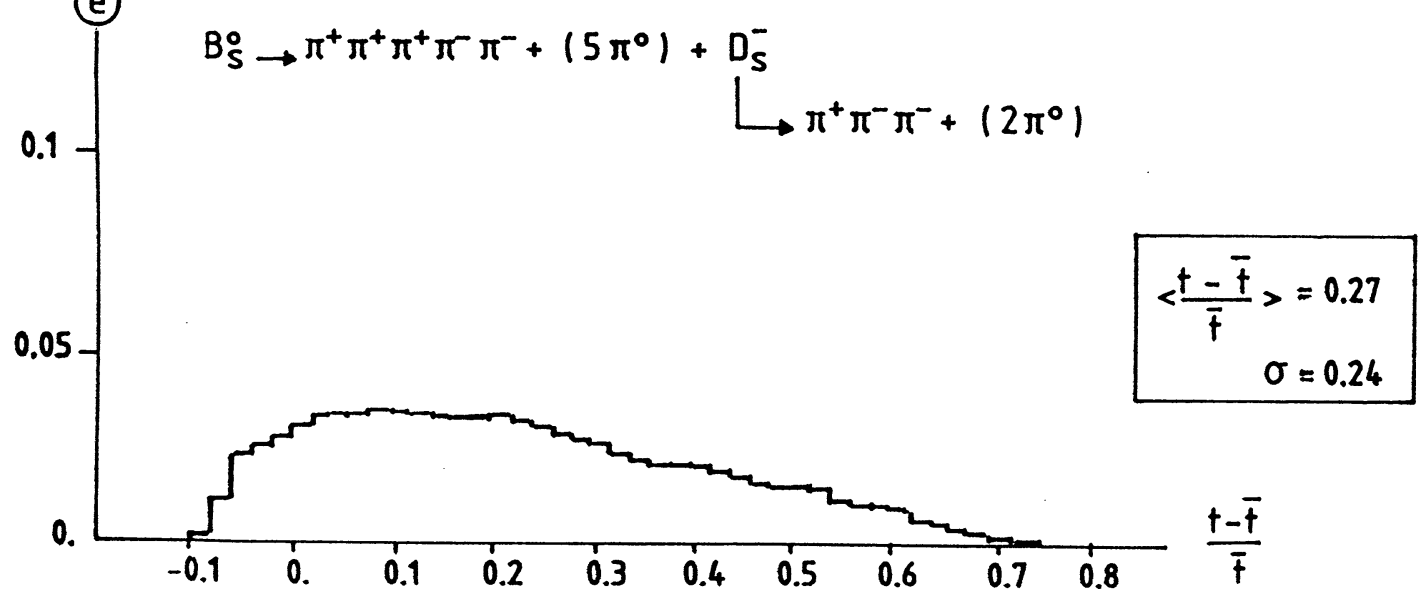

Fig. 2 

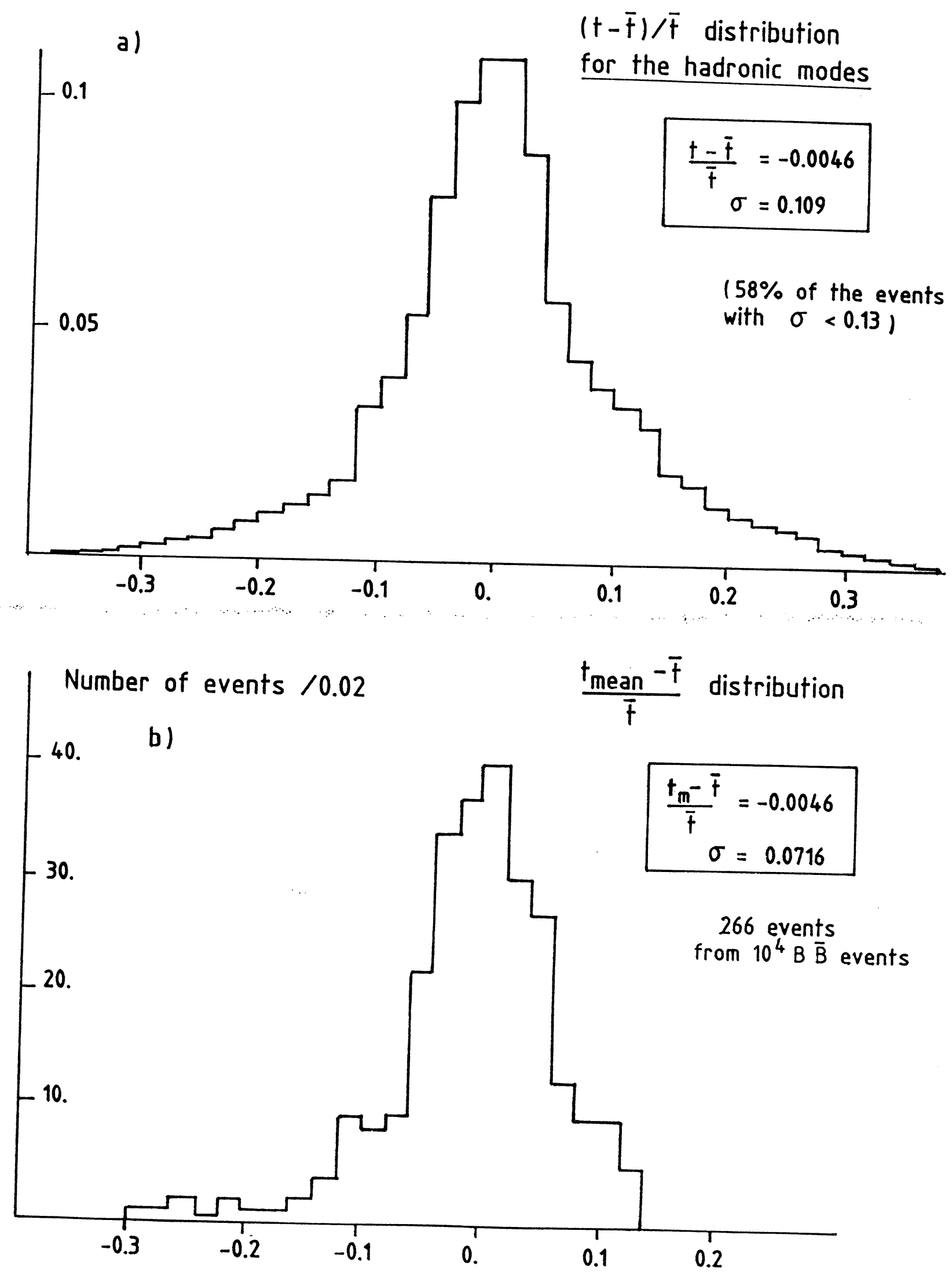

Fig. 3 


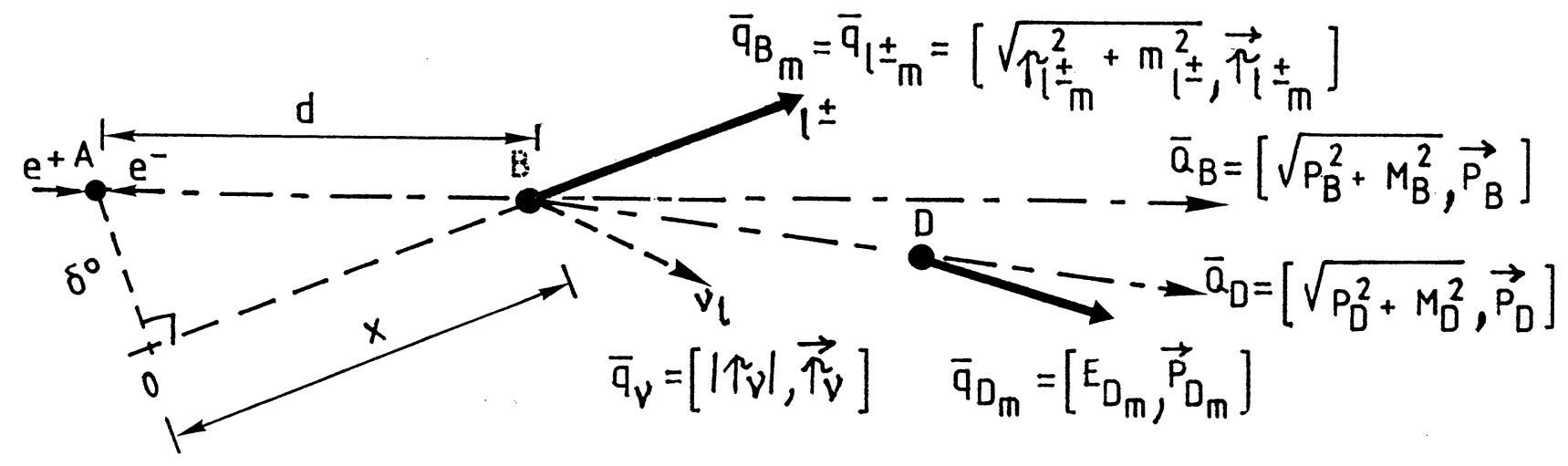

Fig. 4 


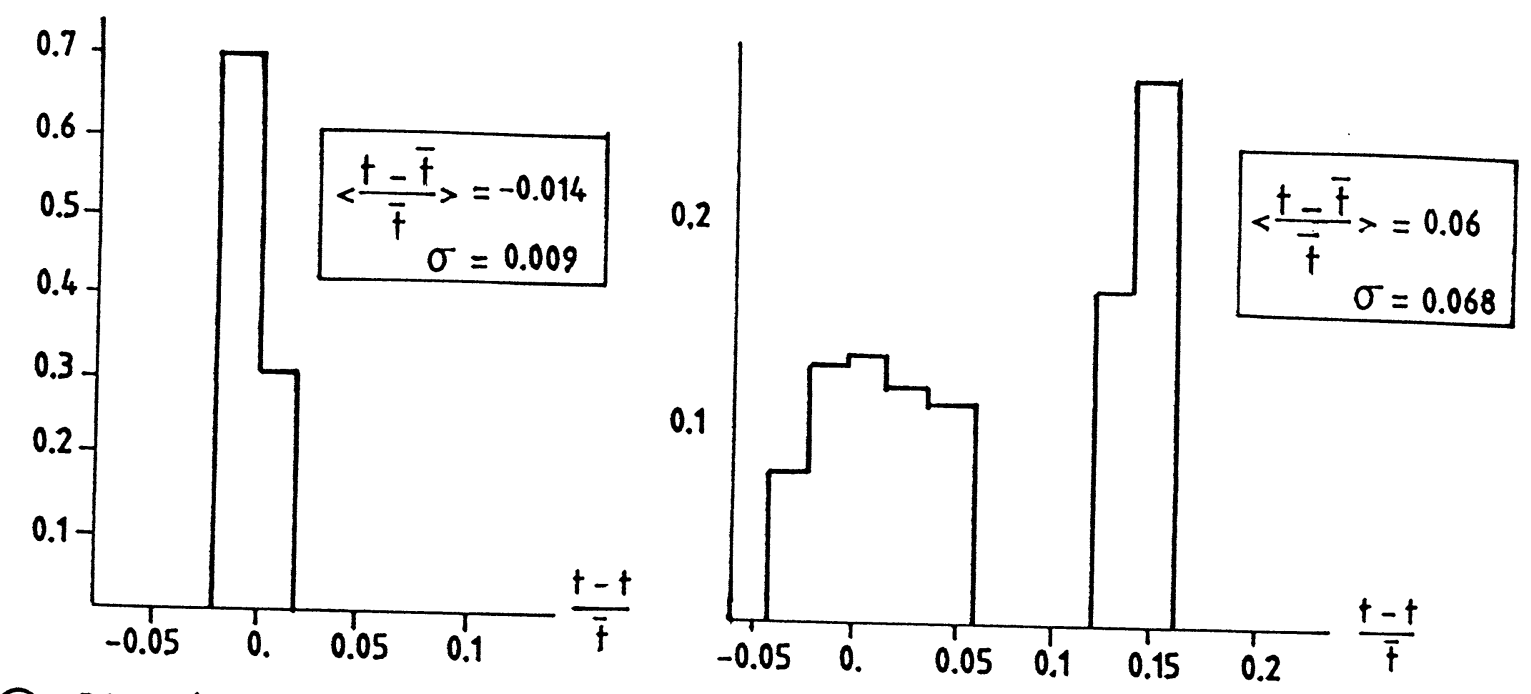

(a): $B_{s}^{\circ} \rightarrow e^{+} v_{e}+D_{s}$

(b) : $B_{S}^{\circ} \rightarrow \mu^{-} v_{\mu}+\stackrel{D_{S}^{*}}{\stackrel{L}{\longrightarrow} D_{s}^{+}+\underset{L}{r} \pi^{+} \pi^{+} \pi^{-}}$

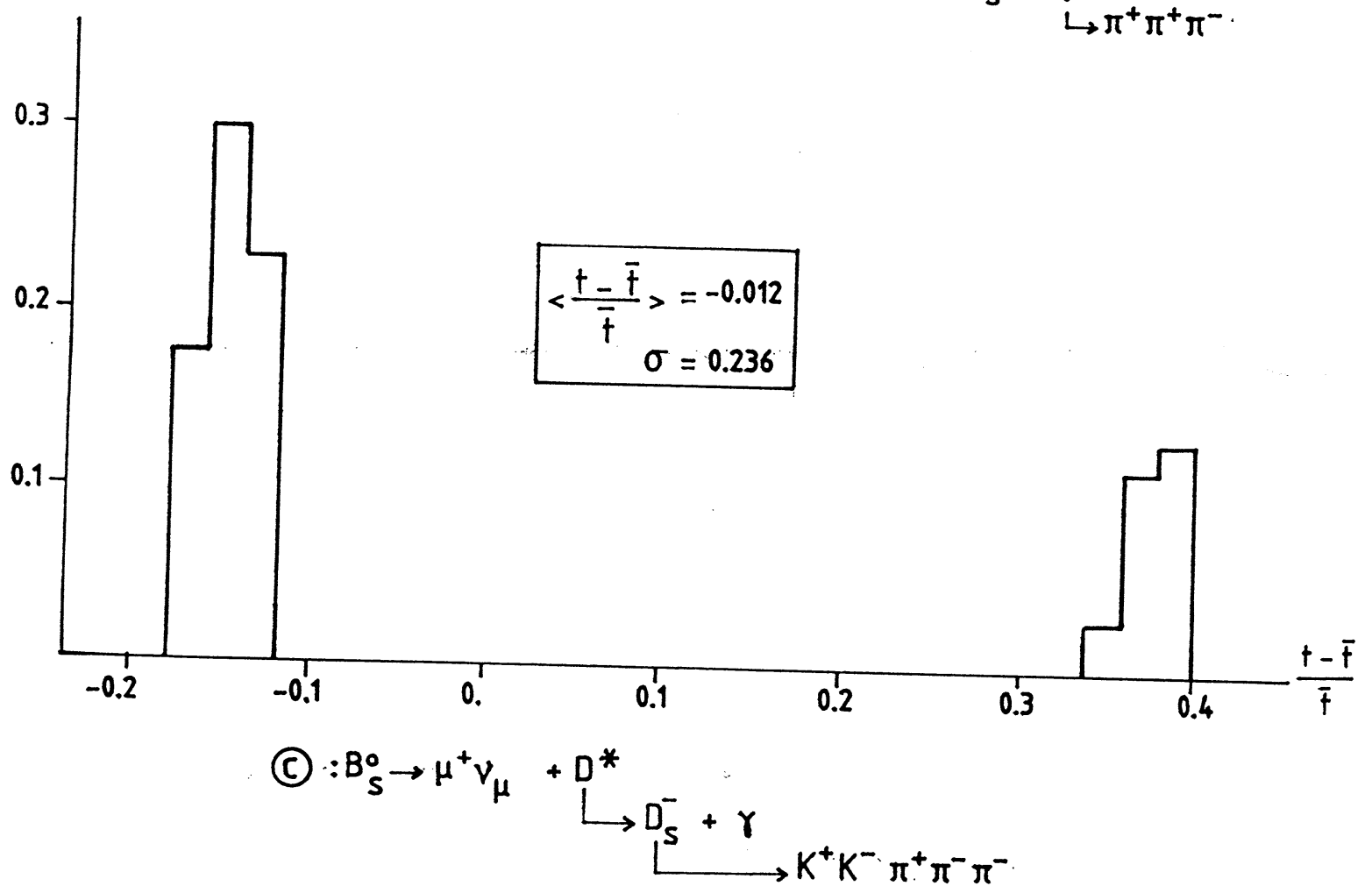

Fig. 5 


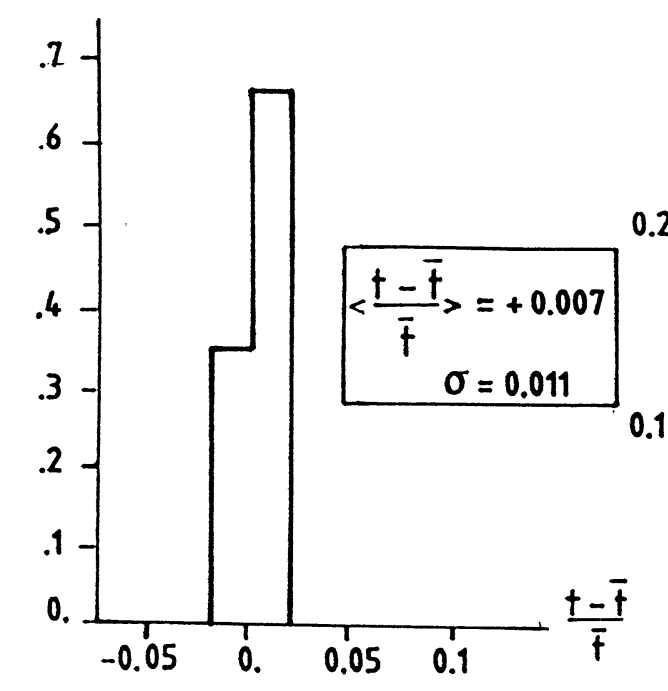

(a) $: B_{S}^{0} \rightarrow e^{-} v_{e}+\stackrel{D_{S}^{*}}{\longrightarrow} \gamma+D_{S}^{+} \rightarrow K^{+} K^{-} \pi^{+} \pi^{+} \pi^{-}+\left(\pi^{\circ}\right)$
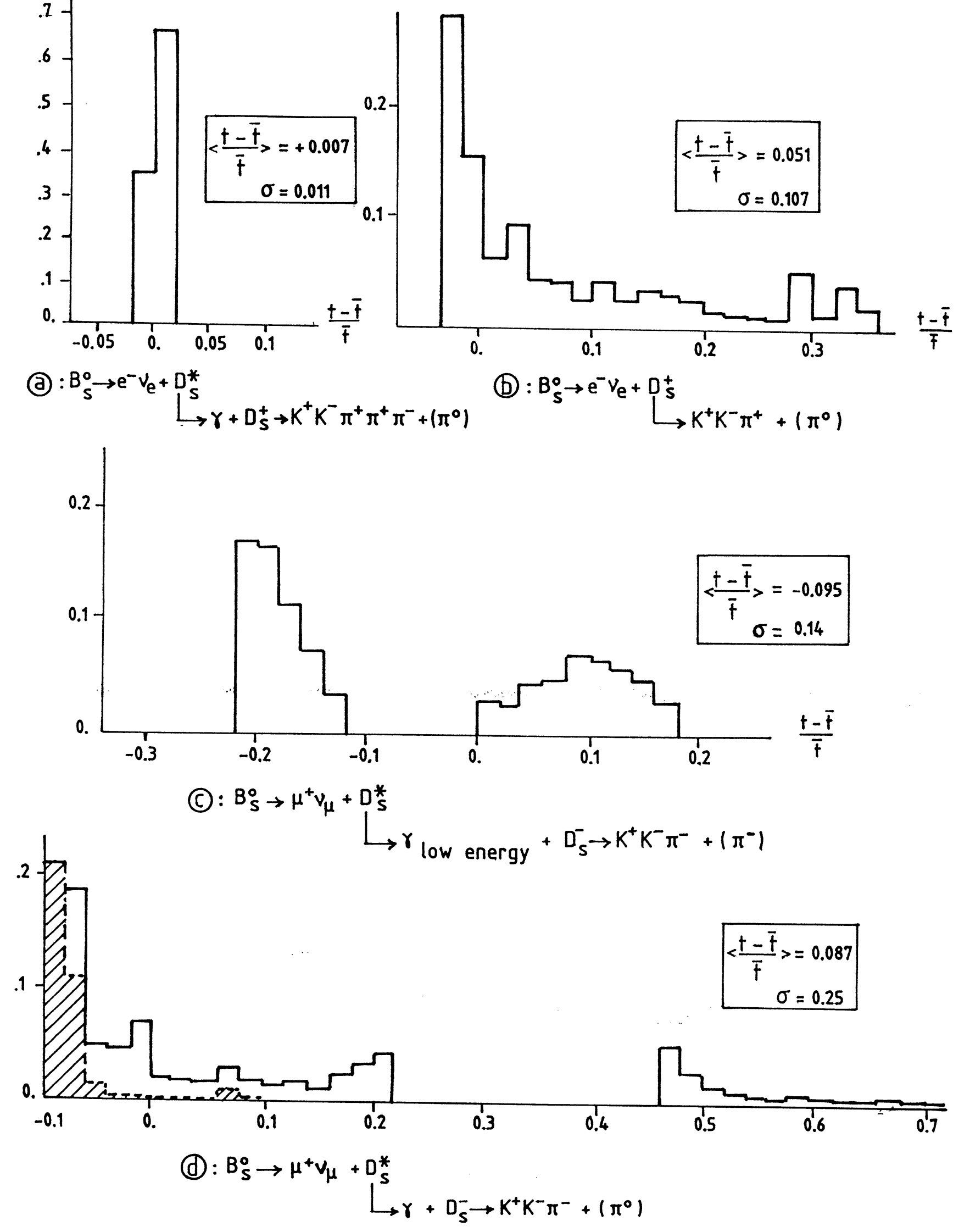

Fig. 6 

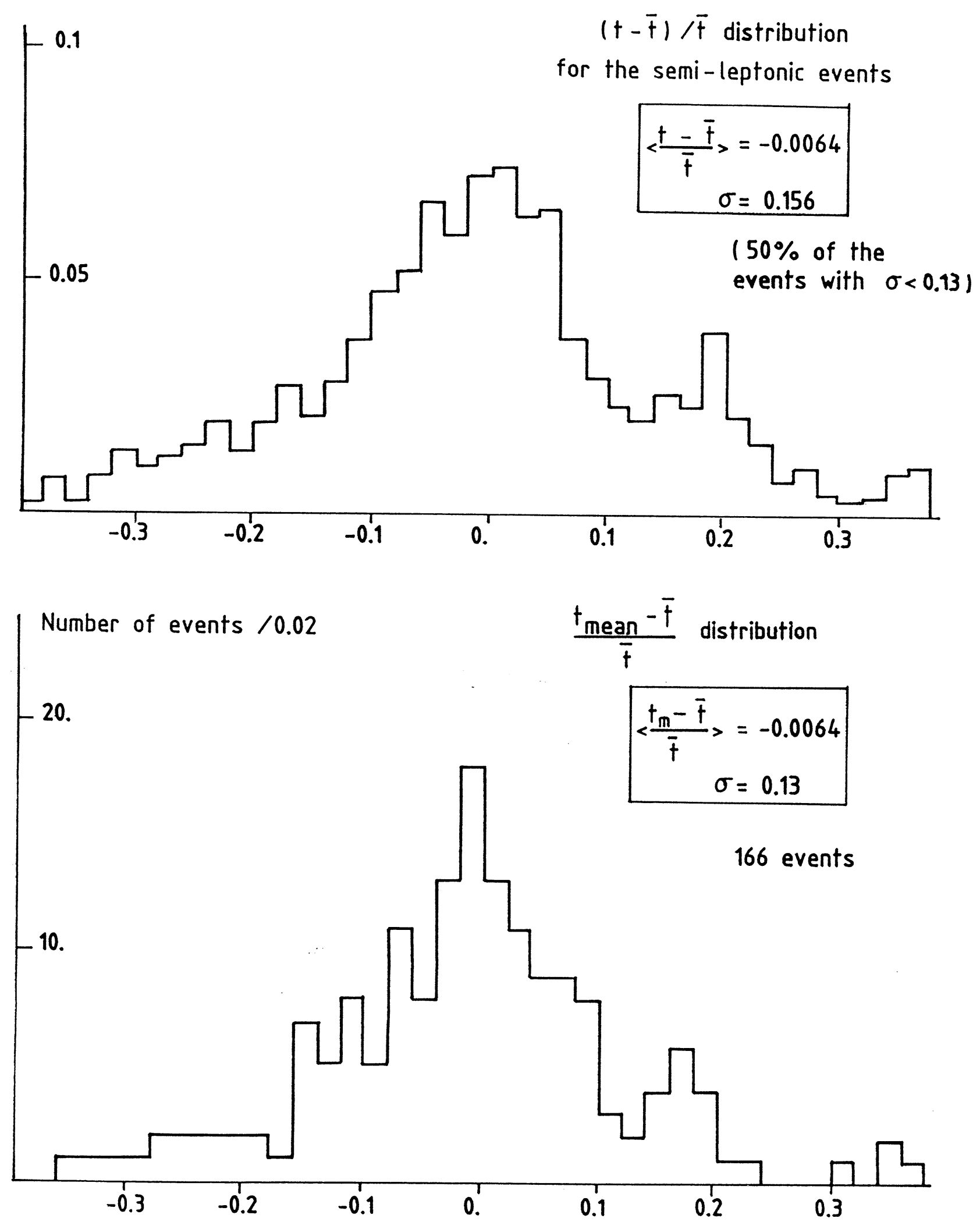

Fig. 7 